\title{
Correction: Upregulation of SNX5 predicts poor prognosis and promotes hepatocellular carcinoma progression by modulating the EGFR-ERK1/2 signaling pathway
}

Qingqing Zhou · Tingting Huang • Zhiyuan Jiang • Chao Ge • Xiaoxia Chen • Lili Zhang • Fangyu Zhao • Miaoxin Zhu • Taoyang Chen · Ying Cui · Hong Li • Ming Yao $\cdot$ Jinjun Li • Hua Tian (D)

Published online: 7 September 2020

(c) The Author(s), under exclusive licence to Springer Nature Limited 2020

Correction to: Oncogene 39, 2140-2155

https://doi.org/10.1038/s41388-019-1131-9

published 9 December 2019

Following publication of this Article the Authors noted that the sequences of shRNA Target of SNX5 in the Supplementary data Table S2 was wrong.
The original sequences of shRNA Target of SNX5 (SNX5-1: GCTTCGCGCCGTAGTCTTA; SNX5-2: TGGC TGCATGCTATGTTGA) was wrong. The right sequences of shRNA Target of SNX5 were: SNX5-1: CCCTCATT GACTATGAGAACT; SNX5-2: GCTTATTATTCCACC TGCTCC.

The Supplementary data Table S2 has been updated. 